\title{
Sampled dynamic holographic memory
}

\author{
Yong Qiao and Demetri Psaltis \\ Department of Electrical Engineering, California Institute of Technology, Pasadena, California 91125
}

Received May 19, 1992

\begin{abstract}
We report the experimental demonstration of a photorefractive dynamic holographic memory that has a simple feedback architecture stabilized through a novel spatial sampling technique. By using dynamic copying to compensate for photorefractive decay, this memory provides nondestructive readout, selective erasure and rewriting, and real-time adaptation of holograms through an arbitrarily large number of exposures.
\end{abstract}

Holograms recorded in photorefractive crystals decay when the crystal is subsequently exposed to light. This effect prevents nondestructive readout of information stored in photorefractive crystals, and it also makes difficult the recording of multiple holograms in the same crystal. Dynamic holographic memories sustain the recorded holograms by dynamically compensating their decay that is due to readout or writing/erasing of other multiplexed holograms. ${ }^{1-4}$ We report here the experimental demonstration of a photorefractive dynamic holographic memory with a simple feedback architecture that is stabilized through a novel spatial sampling technique.

The dynamic memory system, shown in Fig. 1, consists of a single photorefractive crystal for the storage of Fourier-transform holograms, a spatial light modulator (SLM) to display input images, and a feedback loop with a liquid-crystal light valve (LCLV). The LCLV performs image amplification and thresholding. The basic idea is to reconstruct the stored weak hologram, amplify and binarize the reconstructed image with the feedback loop, and rejuvenate the stored hologram by rewriting it with the amplified reconstructed image as the signal beam. Both the input and the signal reconstructed from the hologram are imaged onto the writing side of the LCLV and read out from the other side.

A basic problem with such a feedback loop ${ }^{5}$ is that it is inherently unstable. Any slight misalignment or perturbation in the optical setup will be amplified by the feedback loop, which results in a smeared image and a smeared hologram. A novel feature of this new memory system is that it is stabilized by sampling the image on the reading side of the LCLV with an array illuminator. We used a $128 \times 128$ element microlenslet array and a $4-f$ system to generate the sampling light spot array. When the reconstructed image is fed back to the writing side of the LCLV, each pixel is magnified to a larger circle by placing the LCLV slightly before the image plane. Therefore any misalignment between the input and feedback images will be corrected as long as the readout sampling spot does not miss the magnified pixel incident on the writing side of the LCLV, as shown in Fig. 2. There is an obvious trade-off in this system between image resolution and tolerance to misalignment errors.

An important property of this dynamic memory is that the relative phases of the stored holograms are maintained during copying. This is true because any of the holograms is recorded and copied with the same reference and signal beams that have a fixed phase relationship. In the case of hologram formation by diffusion only (such as for the $\mathrm{BaTiO}_{3}$ crystal used in our experiment), the consequence is that the hologram will always remain phase locked. This property is crucial for the realization of selective memory erasure and holography-based optical neural networks, where the phases of the holograms must be accurately controlled.

Multiple holograms are recorded in the system of Fig. 1 by changing the angle of the reference beam with a mirror mounted on a motorized rotary stage. These holograms are dynamically enhanced by scanning the angle of the reference beam, sequentially reading out and rejuvenating each stored hologram, and repeating this cycle many times.

A simplified diagram of the dynamic memory loop is shown in Fig. 3 , where $x_{i}$ and $y_{i}$ represent the field magnitudes of the $i$ th image pixel at the input and output of the LCLV, respectively. $A$ is the field magnitude of the reference beam, and $\alpha_{1}$ and $\alpha_{2}$ account for losses in the feedback loop. The crystal is oriented so that the optical signal reconstructed by the reference is in phase with the writing signal beam. Such a geometry plus the phase-locking property of this system allow us to use only the magnitudes of

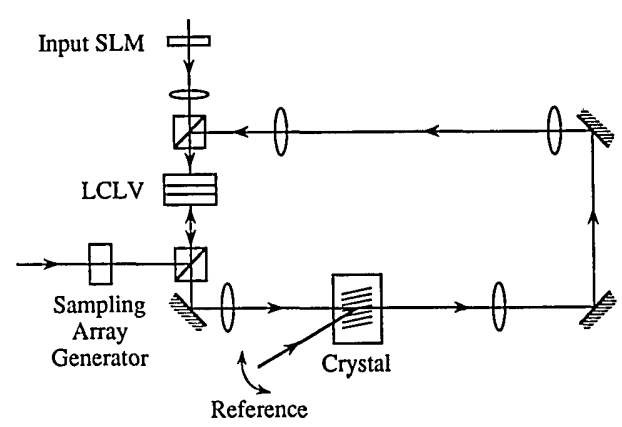

Fig. 1. Schematic diagram of the sampled dynamic holographic memory. 


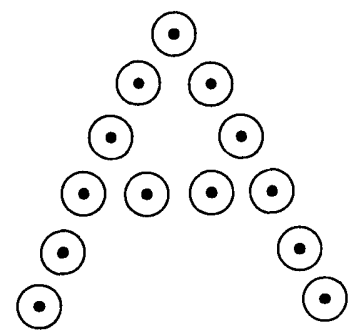

Fig. 2. Illustration of the spatial sampling technique for stabilizing the feedback loop, in which the small dots represent the sampling points on the reading side of the LCLV and the large circles represent the magnified pixels of the feedback image.

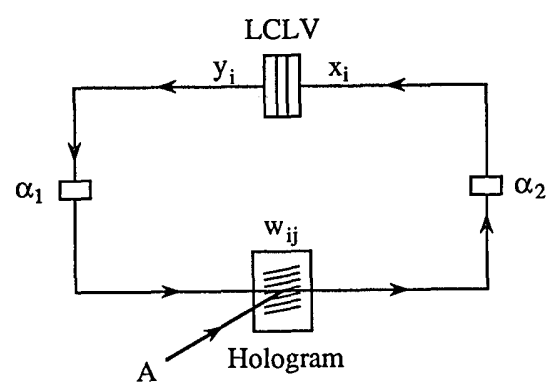

Fig. 3. Simplified system diagram.

all the optical signals for the analysis, without considering their phases. ${ }^{2}$ In the following analysis, we also ignore the two-wave coupling effect in the crystal. Photorefractive holograms written by coupled beams have been treated in detail, ${ }^{6}$ and this effect can be incorporated in our analysis in a straightforward way. Let $w_{i j}$ be the amplitude diffraction efficiency of the grating corresponding to the $i$ th pixel on the LCLV and the $j$ th plane-wave reference. The response time of the LCLV (approximately $20 \mathrm{~ms}$ ) is much faster than that of the $\mathrm{BaTiO}_{3}$ crystal (approximately $3 \mathrm{~s}$ with the light levels used in the experiment). Therefore the signal in the loop (i.e., the output of the LCLV) will first quickly reach its steady state before there is an appreciable change in the hologram recorded in the crystal. The steady state that the signal in the loop will arrive at depends on the initial diffraction efficiency $w_{i j}(0)$, as shown in the following.

Let the nonlinear input-output characteristic of the LCLV be described by $y_{i}=G\left[x_{i}\right]$, which can be approximated by a piecewise linear function

$$
G[u] \approx \begin{cases}0 & u \leq u_{0}, \\ \left(u-u_{0}\right) g & u_{0} \leq u \leq u_{1}, \\ y_{\text {sat }} \equiv\left(u_{1}-u_{0}\right) g & u \geq u_{1},\end{cases}
$$

where $g$ is the gain in the linear region and $u \geq 0$. The magnitude of the optical field incident upon the LCLV is

$$
x_{i}=\alpha_{2}\left[\alpha_{1} y_{i}+A w_{i j}(0)\right] .
$$

Relations (1) and (2) are illustrated in Fig. 4, where we have assumed that $\alpha_{1} \alpha_{2} g>1$. Equation (2) is plotted for two cases: $\alpha_{2} A w_{i j}(0)<u_{0}$ [curve (a)] and $\alpha_{2} A w_{i j}(0)>u_{0}$ [curve (b)]. For case (a) there are three steady states, but only two of them, the on state $y_{i}=y_{\text {sat }}$ and the off state $y_{i}=0$, are stable. In this case, the $i$ th pixel will remain in the off state, since the initial condition for dynamic copying is $\left[x_{i}(0), y_{i}(0)\right]=\left[\alpha_{2} A w_{i j}(0), 0\right]$, which is itself the off state. For case (b), there is only one steady state, i.e., the on state $y_{i}=y_{\text {sat }}$. Thus the $i$ th pixel will always go to the on state. To summarize, the condition for switching on the $i$ th pixel on the LCLV is $\alpha_{2} A w_{i j}(0)>u_{0}$, which simply means that the light amplitude incident upon the writing side of the LCLV, which results from diffraction off the corresponding grating, must exceed the threshold $u_{0}$ of the LCLV.

In this system the cyclical readout and enhancement of the stored holograms lead to a self-driven incremental recording. It has been shown ${ }^{7,8}$ that, with incremental recording, the angularly multiplexed holograms can reach the same final diffraction efficiency without following an exposure schedule. ${ }^{9,10}$ A useful feature of the self-driven incremental recording is that there is no need to provide repeatedly external writing signals during each exposure. Rather, the memory loop itself generates the writing signals by tuning to the appropriate angles of the reference beam, which is a great simplification for practical applications. The final diffraction efficiency of each hologram scales as $1 / R^{2}$ (where $R$ is the number of reference beams), and it does not depend on the number of exposures used to form the initial holograms (before copying).

The dynamics of photorefractive holograms in this system, if we assume that hologram formation is diffusion dominated and that the reference beam is much stronger than the signal beam, can be described by ${ }^{2}$

$$
\tau_{t} \mathrm{~d} w_{i j} / \mathrm{d} t=-w_{i j}+c \alpha_{1} y_{\text {sat }} / A,
$$

where $\tau_{t}$ is the photorefractive time constant and $c$ is a unitless parameter determined by the crystal properties and the recording condition. Strictly speaking, Eq. (3) is valid only for weak gratings. However, $w_{i j}$ scales as $1 / R,{ }^{1}$ and it becomes small when $R$ is large. In this context, Eq. (3) is almost exact. From Eq. (3), we find that the steady-state amplitude diffraction efficiency for $R$ recorded holograms is $c \alpha_{1} y_{\text {sat }} /(A R)$. Using this value for $w_{i j}(0)$ in the switching condition, we obtain the upper bound for the number of angularly multiplexed holograms: $R<c \alpha_{1} \alpha_{2} y_{\text {sat }} / u_{0}$. For ex-

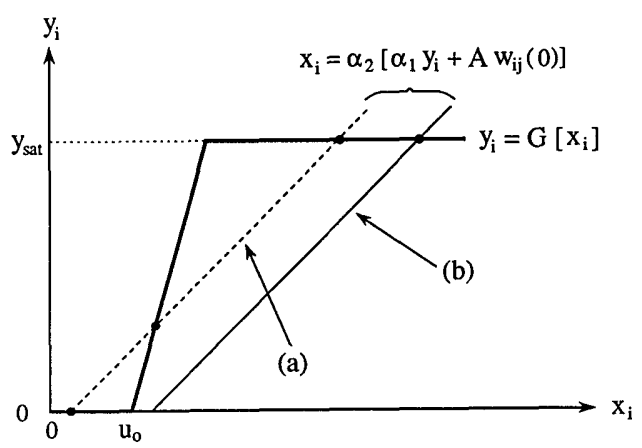

Fig. 4. Steady-state behavior of the dynamic memory. Curve (a), $\alpha_{2} A w_{i j}(0)<u_{0}$; curve (b), $\alpha_{2} A w_{i j}(0)>u_{0}$. 


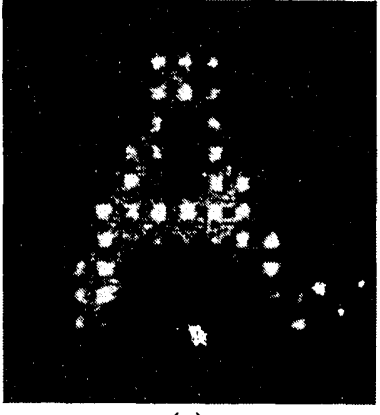

(a)

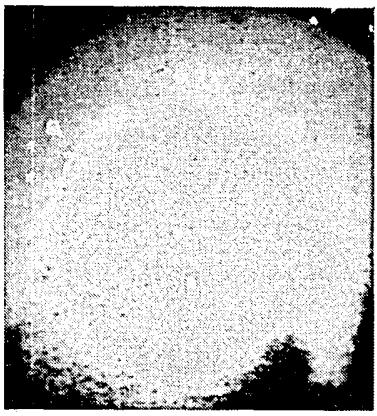

(b)
Fig. 5. Examples of images dynamically stored in the crystal: (a) with sampling, (b) without sampling. (a)

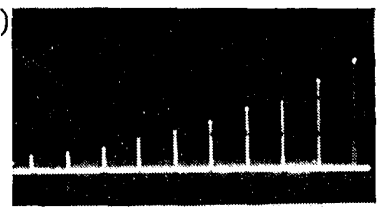

(c)

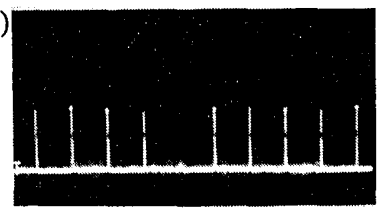

(e)

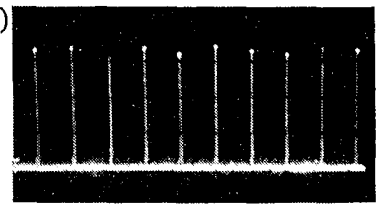

(b)

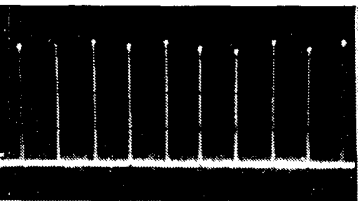

(d)

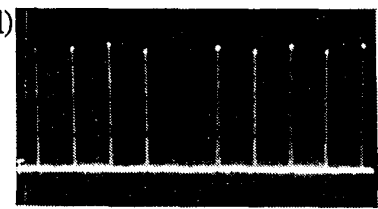

Fig. 6. Experimental results: (a) the diffracted signals of ten angularly multiplexed holograms recorded with equal exposure, (b) the equalized holograms after dynamic copying, (c) selective erasure of the fifth hologram, (d) the other nine holograms rejuvenated by dynamic copying after being partially erased during selective erasure, (e) rewriting of the fifth hologram.

ample, if we assume that $c=1, \alpha_{1}=\alpha_{2}=0.9$, and $y_{\text {sat }} / u_{0}=10^{3}$, the maximum value of $R$ is 810 .

In the experimental apparatus, a $5 \mathrm{~mm} \times$ $5 \mathrm{~mm} \times 5 \mathrm{~mm} \mathrm{BaTiO}{ }_{3}$ crystal with its $c$ axis oriented $45^{\circ}$ from the cut face was used, and the writing beams $(\lambda=514.5 \mathrm{~nm})$ were extraordinary polarized. The stabilization of the feedback loop is demonstrated in Fig. 5. Figure 5(a) shows the steady-state hologram when the initial one was recorded with a single exposure of the letter $\mathrm{A}$ and the sampling was used. The quality of the stored image remained virtually unchanged during the dynamic copying. Figure 5(b) shows the same thing except that the sampling was removed. For the unsampled case, careful alignment simply slowed the transition from a recognizable image to the smeared final state.

A series of experiments were performed to demonstrate the capabilities of dynamic enhancement/ equalization and selective erasure/rewriting of this system. Ten angularly multiplexed image holograms were first recorded with equal exposure, and their diffracted signals were measured with an oscilloscope [shown in Fig. 6(a)]. As expected, the diffraction efficiencies are highly nonuniform. Dynamic copying was then performed for 27 cycles until these holograms were finally equalized and enhanced [Fig. 6(b)]. Next the fifth hologram was erased by introducing a 180-deg phase shift in the corresponding reference beam with an electrically controlled liquid-crystal phase retarder and writing a hologram that is out of phase with the one to be erased. The measured diffracted signals from the stored holograms are illustrated in Fig. 6(c), which shows that the fifth hologram was almost completely erased and the other nine holograms were also partially erased. Subsequent dynamic copying rejuvenated the other nine holograms while the fifth hologram was completely erased because it was too weak to satisfy the switching condition [Fig. 6(d)]. Finally, a new hologram was rerecorded at the fifth position, and all the holograms were again dynamically equalized [Fig. 6(e)]. The final diffraction efficiency of each hologram is approximately $0.12 \%$.

We thank Hsin-Yu Li for his help and many discussions. This research is supported by the Defense Advanced Research Projects Agency and the U.S. Air Force Office of Scientific Research.

\section{References}

1. D. Brady, K. Hsu, and D. Psaltis, Opt. Lett. 15, 817 (1990)

2. Y. Qiao, D. Psaltis, C. Gu, J. Hong, P. Yeh, and R. R. Neurgaonkar, J. Appl. Phys. 70, 4646 (1991).

3. H. Sasaki, Y. Fainman, J. E. Ford, Y. Taketomi, and S. H. Lee, Opt. Lett. 16, 1874 (1991).

4. S. Boj, G. Pauliat, and G. Roosen, Opt. Lett. 17, 438 (1992).

5. K.-Y. Hsu, H.-Y. Li, and D. Psaltis, Proc. IEEE 78, 1637 (1990).

6. J. H. Hong and R. Saxena, Opt. Lett. 16, 180 (1991).

7. R. McRuer, J. Wilde, L. Hesselink, and J. Goodman, Proc. Soc. Photo-Opt. Instrum. Eng. 881, 192 (1988).

8. Y. Taketomi, J. E. Ford, H. Sasaki, J. Ma, Y. Fainman, and S. H. Lee, Opt. Lett. 16, 1774 (1991).

9. K. Bløtekjaer, Appl. Opt. 18, 57 (1979).

10. D. Psaltis, D. Brady, and K. Wagner, Appl. Opt. 27, 1752 (1988). 